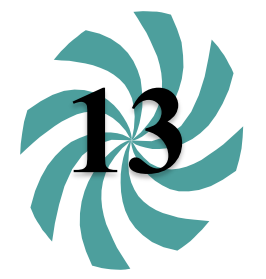

Tecnociencia, Vol. 22, $\mathrm{N}^{\circ} 2: 245-257$

julio-diciembre 2020

\title{
IMPORTANCIA DEL RECURSO AGUA PARA EL CONSUMO DOMÉSTICO EN EL DISTRITO DE LA CHORRERA DE LA REPÚBLICA DE PANAMÁ
}

\author{
Carlos Antonio Villarreal \\ Universidad de Panamá, Facultad de Ciencias Naturales Exactas y Tecnología; \\ Departamento de Ciencias Ambientales; Escuela de Biología. \\ E-mail: Calito.villa@yahoo.com
}

\section{RESUMEN}

En este artículo presentamos la importancia del agua para el consumo doméstico en el distrito de La Chorrera, república de Panamá. El crecimiento poblacional, el rápido desarrollo económico, la urbanización, los efectos adversos del cambio climático global y el propio deterioro ambiental, continúan aumentando la presión sobre los recursos hídricos, de tal manera que se registran ya condiciones de aparente escasez, particularmente en zonas rurales y periurbanas con baja o inexistente inversión pública en seguridad hídrica. Se observa que, en cuanto al recurso del agua, ya hay manifestaciones en todo el territorio nacional, en las distintas provincias, distritos, corregimientos, lugares o comunidades, sobre la falta del vital recurso por distintas razones. Para el desarrollo del estudio se toma como técnica, la elaboración de encuestas estructuradas a una muestra al azar de hombres y mujeres adultos del distrito de La Chorrera. A través de la cual se espera conocer los diferentes mecanismos que utiliza la población para la obtención del agua de uso doméstico en la comunidad. Se recomienda establecer políticas nacionales que vayan enfocando el cambio en la mentalidad de la población con la importancia de cuidar y hacer uso racional del agua.

\section{PALABRAS CLAVES}

Recurso, hídrico, contaminación, sostenible, doméstico. 


\title{
IMPORTANCE OF THE WATER RESOURCE FOR DOMESTIC CONSUMPTION IN THE CHORRERA DISTRICT OF THE REPUBLIC OF PANAMA
}

\begin{abstract}
In this article we present the importance of water for domestic consumption in the district of La Chorrera, republic of Panama. Population growth, rapid economic development, urbanization, adverse effects of global climate change and environmental deterioration itself continue to increase pressure on water resources, thus already registering conditions of apparent scarcity, particularly in rural and periurban areas with low or no public investment in water security. It is noted that, with regard to the use of water, there are already demonstrations throughout the national territory, in the different provinces, districts, corrections, places or communities, about the lack of vital resource for different reasons. For the development of the study is taken as a technique, the elaboration of structured surveys to a random sample of adult men and women of the district of La Chorrera. Through which you expect to know the different mechanism that the population uses to obtain water for domestic use in the community. It is recommended to establish national policies that focus the change on the mentality of the population with the importance of caring for and making rational use of water.
\end{abstract}

\section{KEY WORDS}

Resource, water, contamination, sustainable, domestic.

\section{INTRODUCCIÓN}

Históricamente el marco legal e institucional del agua en Panamá ha sido responsabilidad de diferentes ministerios e instituciones que han tenido derecho sobre esta con el pasar de las décadas.

A inicio del siglo, se establece un régimen administrativo especial para el manejo de los recursos hídricos a partir de la Ley 44 del 5 de agosto de 2002. En 2005, se adicionan los delitos contra el ambiente en el Código Penal, a partir de la Ley 5 del 28 de enero. En 2006 se crea la Autoridad de los Recursos Acuáticos de Panamá como responsable del manejo de los recursos hídricos; en 2015 se crea el Ministerio de Ambiente, también con la finalidad de gestionar el buen manejo de los recursos hídricos, y el 23 de agosto de 2016 se funda el Consejo 
Nacional del Agua (CONAGUA), como ente responsable de la ejecución del Plan Nacional de Seguridad Hídrica 2015-2050.

Diariamente una persona debe consumir 80 litros de agua para suplir sus necesidades y en Panamá, se consume de 160 a 200 litros de agua, lo que es realmente un despilfarro. Es importante la conservación de las cuencas, del cuidado de los ríos y de los bosques de galería, pues en la ciudad de Panamá, habitan más de 200 mil personas que no tienen agua potable o que presenta deficiencia, sin embargo, esto se debe a que han invadido terreno. Antes del 2015 se debe bajar a la mitad, la deficiencia que tenemos en cobertura.

Se observa que, en cuanto al recurso del agua, ya hay manifestaciones en todo el territorio nacional, en las distintas provincias, distritos, corregimientos, lugares o comunidades, sobre la falta del vital recurso por distintas razones.

No hay suficientes fuentes de agua, están agotadas. Las fuentes están contaminadas por diversas actividades humanas. Hay que buscar aguas subterráneas con perforaciones de pozos, y ya en algunas zonas ni siquiera hay aguas subterráneas.

El desabastecimiento de agua en el distrito de La Chorrera, debido a la mala utilización del recurso y al aumento demográfico trae como consecuencia la urgente necesidad de perforación de nuevos pozos y el bajo nivel que registran los actuales pozos. Ante tales casos, se ha establecido un programa de construcción de acueductos rurales.

En la actualidad, los problemas de escasez, falta de mecanismos de obtención y contaminación del agua generan conflictos sociales a diferentes escalas: Entre comunidades, provincias, y los habitantes del país en general. Si los problemas relacionados con el agua no son atendidos de forma oportuna, es inminente el riesgo de que los conflictos escalen y la lucha por la posesión del agua convierta a zonas del país en campos de batalla. 
En consecuencia, resulta necesario realizar un estudio serio sobre este problema, en aras de contribuir a generar soluciones viables a los problemas generados por la ausencia de una política sustentable de gestión de agua en Panamá.

\section{MATERIALES Y MÉTODOS}

La investigación se enmarcó en un diseño Descriptivo-Explicativo, utilizando el paradigma cuantitativo más que cualitativo, por cuanto lo que se deseaba conocer eran los mecanismos que desarrolla la población para obtener el recurso agua para el consumo y demás actividades domésticas.

Durante la fase de recabar los datos de la investigación, se tuvo la oportunidad de hacer los primeros contactos directos con miembros de la comunidad y de autoridades del distrito, realizando las visitas semanales por un periodo de un mes. Permitiendo así la elaboración y posterior desarrollo del instrumento de recolección establecido.

Para lograr la participación de la comunidad en facilitar la información se utilizaron diversos mecanismos como son: Encuestas de opinión y entrevistas, esto a su vez permitió abrir un canal de información entre la población y el encuestador.

Se aplicaron 30 encuestas estructuradas a una muestra al azar de hombres y mujeres en tres sectores (Río Congo, Santa Cruz y El Arado) pertenecientes al corregimiento del Arado, distrito de La Chorrera. Los cuales concentran una población aproximada de 775 habitantes. A razón de 10 encuestas por sector. La misma se elaboró con el siguiente formato: 


\section{Formato de Encuesta}

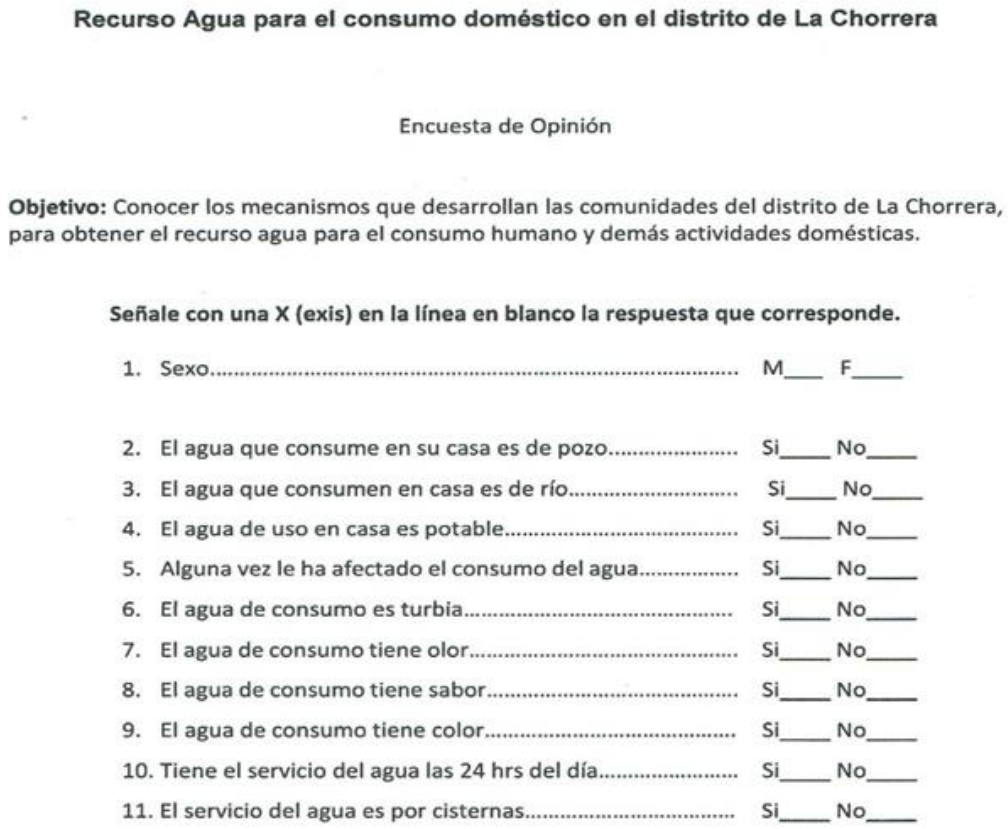

Agradeciéndole por su aporte al estudio.

Encuestador: Carlos A. Villarreal

\section{RESULTADOS Y DISCUSIÓN}

Presentamos los análisis de los resultados obtenidos a través de la recolección de datos a la población del distrito de La Chorrera. 
cuadro 1. Sexo de la población encuestada

\begin{tabular}{lcc}
\hline \multicolumn{3}{c}{ Sexo de la población encuestada } \\
\hline & Cantidad & Porcentaje \\
\hline Masculino & 11 & $37 \%$ \\
Femenino & 19 & $63 \%$ \\
\hline
\end{tabular}

Gráfica 1: Sexo de la persona encuestada

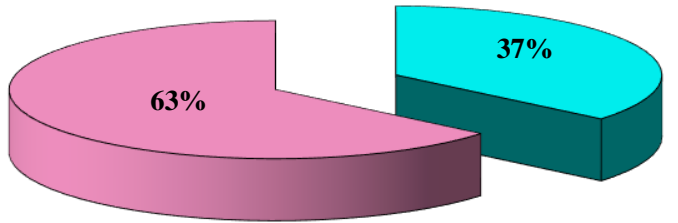

口Masculino

口Femenino

A pesar que se quiso entrevistar la misma cantidad de mujeres y hombres, como se observa hubo una mayor participación de las mujeres que de los hombres. Éstas se mostraron más abiertas y receptivas a responder las preguntas. Por lo que se observa un 63\% de la población femenina y un $37 \%$ de la población masculina.

Cuadro 2. El agua que consume en su casa es de pozo

\begin{tabular}{lcc}
\hline & \multicolumn{3}{c}{ El agua que consume en casa es de pozo } \\
\hline & Cantidad & Porcentaje \\
\hline $\mathrm{Si}$ & 5 & $17 \%$ \\
$\mathrm{No}$ & 25 & $83 \%$ \\
\hline
\end{tabular}

Gráfica $N^{\circ}$ 2: El aguaque consume en casa es de pozo

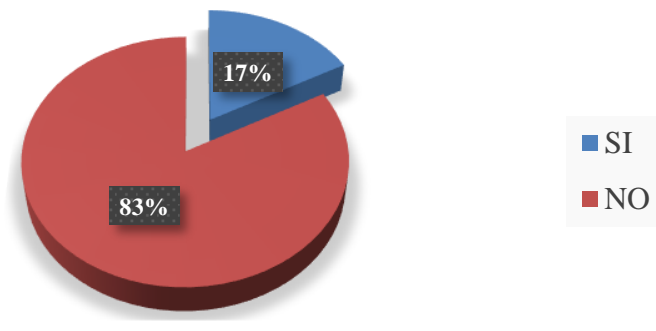


La población estudiada manifiesta en un $17 \%$ que tiene como mecanismo de obtener agua para su consumo de un pozo artesanal, mientras que el $83 \%$ cuenta con otras formas de obtención del agua para su consumo en los hogares.

Cuadro 3. El agua que consumen en casa es de río

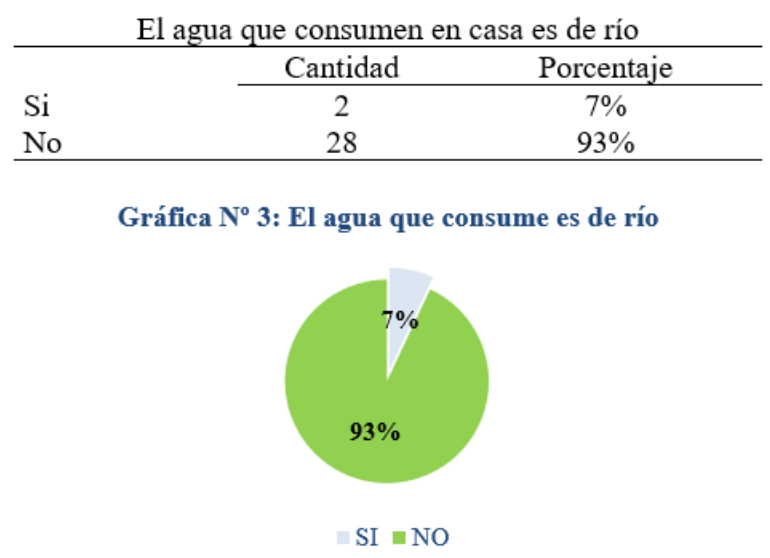

El 7\% de los consultados manifiestan que obtienen el agua de consumo doméstico del río, mientras que el $93 \%$ la abtienen a través de otros mecanismos.

Cuadro 4. El agua de uso en casa es potable

\begin{tabular}{lcc}
\hline & \multicolumn{3}{c}{ El agua de uso en casa es potable } \\
\hline & Cantidad & Porcentaje \\
\cline { 2 - 3 } $\mathrm{Si}$ & 23 & $77 \%$ \\
No & 7 & $23 \%$ \\
\hline
\end{tabular}

Gráfica $\mathrm{N}^{\circ}$ 4: El agua de uso en casa es potable

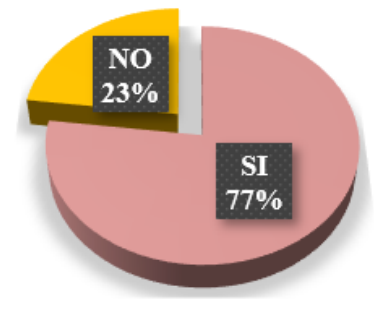

Tecnociencia, Vol. 22, $N^{\circ} 2$ 
Según los datos obtenidos el $77 \%$ de la población objeto de estudio cuenta con el servicio de agua potable en sus viviendas, por otro lado, un $23 \%$ aún no cuenta con dicho servicio para el consumo en sus viviendas.

Cuadro 5. Alguna vez le ha afectado el consumo del agua

\begin{tabular}{lcc}
\hline \multicolumn{3}{c}{ Alguna vez le ha afectado el consumo del agua } \\
\cline { 2 - 3 } $\mathrm{Si}$ & Cantidad & Porcentaje \\
No & 26 & $87 \%$ \\
\hline \multicolumn{2}{c}{ Gráfica $N^{\circ}$ 5: Alguna vez le ha afectado el consumo del agua }
\end{tabular}

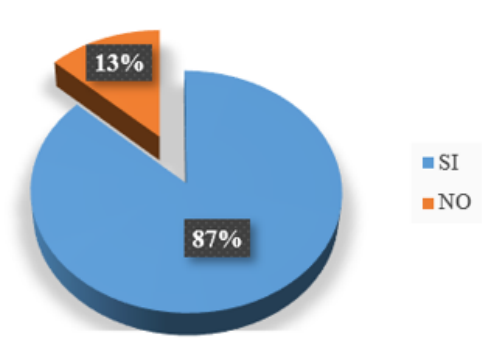

A través de la información obtenida, el $87 \%$ de la población estudiada manifiesta haber sido afectada por la falta de agua para el consumo diario, mientas que un $13 \%$ manifiesta que no se ha visto afectada por esta situación.

Cuadro 6. El agua de consumo es turbia

\begin{tabular}{lcc}
\hline \multicolumn{3}{c}{ El agua de consumo es turbia } \\
\hline & Cantidad & Porcentaje \\
\cline { 2 - 3 } $\mathrm{Si}$ & 14 & $47 \%$ \\
$\mathrm{No}$ & 16 & $53 \%$ \\
\hline
\end{tabular}

Gráfica $N^{\circ}$ 6: El agua de consumo es turbia

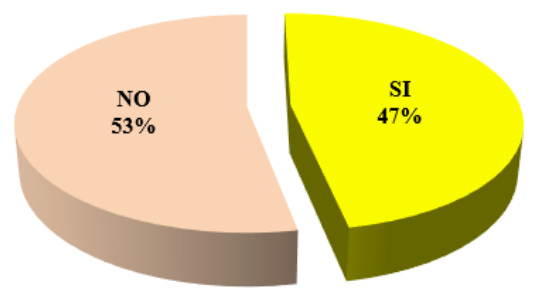

$m \mathrm{SI}=\mathrm{NO}$ 
Según los encuestados un $47 \%$ ha observado la turbiedad que presenta el agua de consumo diario al llegar a sus viviendas, un 53\% no ha observado que el agua de consumo diario presente turbiedad.

Cuadro 7. El agua de consumo tiene olor

\begin{tabular}{lcc}
\hline \multicolumn{3}{c}{ El agua de consumo tiene olor } \\
\hline & Cantidad & Porcentaje \\
\cline { 2 - 3 } Si & 3 & $10 \%$ \\
No & 27 & $90 \%$ \\
\hline
\end{tabular}

Gráfica $N^{\circ} 7$ : El agua de consumo tiene olor

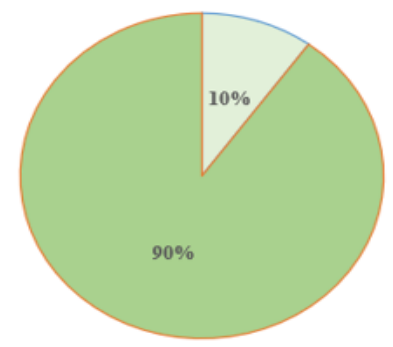

$\square$ SI $\square$ NO

El 10\% de la población tomada para el estudio, considera que el agua de consumo diario presenta olor, mientras que el $90 \%$ de la población considera que no ha presentado ningún olor.

Cuadro 8. El agua de consumo tiene sabor

\begin{tabular}{lcc}
\hline \multicolumn{3}{c}{ El agua de consumo tiene sabor } \\
\hline & Cantidad & Porcentaje \\
\cline { 2 - 3 } Si & 6 & $20 \%$ \\
No & 24 & $80 \%$ \\
\hline
\end{tabular}

Gráfica $N^{\circ}$ 8: El agua de consumo tiene sabor

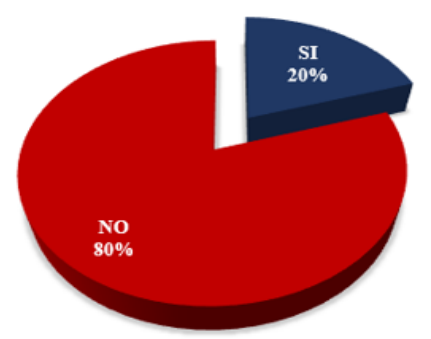

Tecnociencia, Vol. 22, $N^{\circ} 2$ 
En ocasiones el agua de consumo presenta un sabor no propio según lo manifiesta un $20 \%$ de la población estudiada, el $80 \%$ no ha sentido ningún sabor al agua de consumo que llega a sus hogares.

Cuadro 9. El agua de consumo tiene color

\begin{tabular}{lcc}
\hline \multicolumn{3}{c}{ El agua de consumo tiene color } \\
\hline & Cantidad & Porcentaje \\
\cline { 2 - 3 } $\mathrm{Si}$ & 13 & $43 \%$ \\
$\mathrm{No}$ & 17 & $57 \%$ \\
\hline
\end{tabular}

GRÁFICA No 9: EL AGUA TIENE COLOR

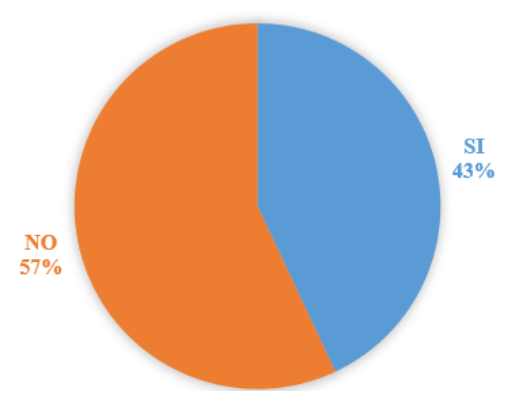

El $43 \%$ de la población ha observado un color no propio del agua de consumo, mientras que el 57\% manifiesta no haber observado color al agua de consumo diario.

Cuadro 10. Tiene el servicio del agua las 24 horas del día

\begin{tabular}{|c|c|c|}
\hline \multicolumn{3}{|c|}{ Tiene el servicio del agua las 24 horas del dí } \\
\hline & Cantidad & Porcentaje \\
\hline $\mathrm{Si}$ & 12 & $40 \%$ \\
\hline No & 18 & $60 \%$ \\
\hline
\end{tabular}

GRÁFICA N ${ }^{\circ} 10$ : TIENE SERVICIO DEL AGUA LAS 24 HORAS DEL DIA

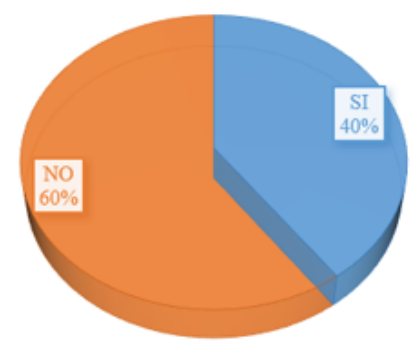


Podemos observar que el $60 \%$ de la población encuestada manifiesta que no cuenta con el servicio de agua potable las 24 horas del día, por el contrario, el $40 \%$ manifiesta que cuenta con dicho servicio.

Cuadro 11. El servicio del agua es por cisternas

\begin{tabular}{lcc}
\hline \multicolumn{3}{c}{ Tiene el servicio del agua las 24 horas del día } \\
\hline & Cantidad & Porcentaje \\
\cline { 2 - 3 } $\mathrm{Si}$ & 12 & $40 \%$ \\
$\mathrm{No}$ & 18 & $60 \%$ \\
\hline
\end{tabular}

Gráfica $\mathrm{N}^{\circ}$ 11: El servicio del agua es por cisterna

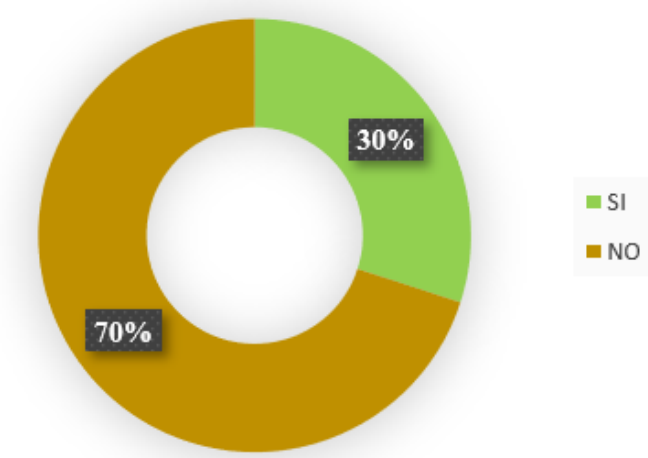

Mientras que el 30\% de la población objeto de estudio manifiesta que recibe el agua de consumo a través de carros cisternas, el $70 \%$ lo recibe a través de otros mecanismos de obtención de agua potable para el consumo diario.

\section{CONCLUSIONES}

En conclusión, el crecimiento demográfico que ha experimentado el sector en las últimas décadas ha generado un aumento de la demanda del recurso agua, muy por encima de las capacidades de los servicios y de la infraestructura de abastecimiento y saneamiento existente, los cuales son insuficiente para dar respuesta a la población. 
Por otro lado, las fuentes de agua están agotadas, debido a diferentes factores que intervienen en el área. Las fuentes están contaminadas por diversas actividades humanas. Hay que buscar aguas subterráneas con perforaciones de pozos, y ya en algunas zonas ni siquiera hay aguas subterráneas.

A pesar de la existencia de una potabilizadoras el problema de la falta de agua en el sector persiste. Esto debido, en algunas ocasiones, a que la misma presenta desperfectos mecánicos por falta de mantenimiento y no pueden suministrar el agua a la población; en algunos casos, tardan hasta un (1) mes para repararlas dependiendo del daño, además el servicio se ve interrumpido cuando las tomas de agua de las potabilizadoras son obstruidas por piedras, tierras, arenas, troncos y desechos, producto de crecidas de ríos que provocan fuertes inundaciones, probablemente relacionado a la gran tasa de deforestación de las cuencas hidrográficas.

\section{REFERENCIAS}

Álvarez, M. A., 2013. Evaluación del consumo en Hogares del Agua potable producida por el método de electrólisis en Ipetí Emberá. Panamá.

Álvarez, M. A. 2011 y 2016. Diagnóstico Socioeconómico de la comunidad de Ipetí Emberá.

Howard, Guy y Jamie Bartram. 2003. Water Engineering and Development Centre, Universidad de Loughborough, RU, Organización Mundial de la Salud, Ginebra, Suiza HO/SDE/WSH/03.02 OMS, Ginebra. La cantidad de agua domiciliaria, el nivel del servicio y la salud. [Online] disponible:

https://www.who.int/water_sanitation health/diseases/WSH03.02.pdf? $\underline{\mathrm{ua}=1}$

López, Campistrous, García, Monier B. y Vázquez, Librada. 2005. Estudio de la obtención electrolítica "in situ" de disoluciones acuosas 
de hipoclorito de sodio. Universidad de Oriente. Tecnología Química. Vol XXV, No 2 .

Tejada, L. M. 2014. Ministerio de Economía y Finanzas. Atlas Social de Panamá. Desigualdades en el acceso y el uso de Agua potable en Panamá. [Online]. En:www.mef.gob.pa/es/informes/Paginas/Atlas$\underline{\text { Social.aspx }}$

Derechos de propiedad intelectual 2016. Consejo Nacional del Agua. Primera Edición, 3000 ejemplares. Este documento debe citarse: Comité de Alto Nivel de Seguridad Hídrica -Plan Nacional de Seguridad Hídrica 2015-2050: Agua para Todos, Panamá Rep. De Panamá.

Ministerio de Salud (MINSA). Decreto Ejecutivo No.1839 del 5 de diciembre de 2014. Gaceta oficial digital No.27678-A del 11 de diciembre del 2014. Unifica el Decreto 40 y Resolución, dictamina nuevo marco regulatorio de JAAR [Online] www.asamblea.gob.pa

Ministerio de Salud (MINSA), Alianza por el Agua (Colaboración). Agencia Española de Cooperación Internacional (AECID - Organismo Financiador). Las Juntas Administradoras de Acueductos Rurales JAAR. Aportando al Derecho Humano al Agua en: panamá.www.alianzasporelagua.org/documentos/gestión_Comunitaria Agua/Panama.pdf

Ley 72 del 23 de diciembre de 2008. Procedimiento especial para establecer o adjudicar las propiedades del territorio de pueblos indígenas que están fuera de comarcas, en cumplimiento del artículo 127 de la Constitución Política de la República de Panamá.

Enciclopedia Medioambiental. El consumo de agua en porcentajes. Consumo de agua per cápita. [Online] disponible:

https://www.ambientum.com/enciclopedia medioambiental/aguas/el/el -consumo-de-agua-en-porcentajes.

Recibido 20 de abril de 2020, aceptado 8 de mayo de 2020. 